\section{Nous avons un décollage! Le lancement de la Compétence par le design au Canada}

La transition vers l'image de marque du Collège royal des médecins et chirurgiens du Canada de la formation médicale axée sur les compétences (FMC), intitulée "Competence By Design (CBD)", débutera le 1er juillet 2019 pour de nombreux programmes de médecine interne, y compris les programmes de médecine interne de base et les programmes généraux de bourses de recherche en médecine interne. Pour de nombreuses personnes qui s'intéressent à la formation médicale postdoctorale, il s'agit d'un voyage vers l'inconnu. Pour faciliter cette transition, voici un bref résumé de la CBME pour ceux qui sont moins familiers avec les changements à venir.

\section{Qu'est-ce qui a inspiré cette transition ?}

Les efforts visant à réformer la conception de l'éducation, d'un système fondé sur des processus à un système axé sur les résultats de la formation ou les compétences, se poursuivent depuis le début des années 20001. L'initiative CanMEDS, adoptée en 1996 et mise à jour en 2005, définit un cadre de compétences solide et reconnu internationalement. Cependant, la mise en œuvre et l'évaluation du cadre de compétences CanMEDS demeurent un défi au sein des structures de formation canadiennes traditionnelles. Le Collège royal a donc créé une structure de la CBME intitulée CBD, qui a mené à une refonte de la conception des programmes détudes et du système d'évaluation pour tous les programmes de formation postdoctorale, dans le but de mettre l'accent sur les résultats de la formation, l'acquisition des compétences et la promotion de la centralité de l'apprentissage2.

\section{Quelles sont les principales différences à prévoir ?}

De nombreux changements sont nécessaires afin d'aligner les programmes de troisième cycle sur les nouvelles exigences de la CDB. Il sagit notamment de :

- Recadrer les jalons du développement à partir des jalons traditionnels fondés sur le temps (1re, 2e et 3e années d'études postdoctorales) jusqu’à ceux décrits par le Collège (Transition vers la discipline, fondations, noyau de la discipline et transition vers la pratique).

- Création d'un programme d'évaluation, mettant l'accent sur les outils d'évaluation en milieu de travail (WBA), afin d'assurer lobservation directe et la documentation des compétences acquises. Le programme WBA a été conçu à l'aide d'activités professionnelles dignes de confiance (AEP) pour saisir les compétences de base (résultats ou compétences) requises par un médecin dans une spécialité donnée.3 Le Collège royal, les universités et les programmes individuels travaillent à créer une plate-forme électronique pour saisir ces évaluations multiples au fil du temps pour chaque stagiaire. Il existe actuellement de nombreuses plates-formes différentes en exploitation dans l'ensemble du pays.

- Création d'un processus visant à promouvoir “ l'évaluation au service de l'apprentissage " et à encourager les stagiaires à réfléchir sur leurs données dévaluation, leur progression et le développement de leurs compétences. Les stratégies à cet égard ont notamment consisté à créer le rôle d'un coach académique.

- Création d'un processus d'examen des données d'évaluation et de promotion équitable du stagiaire tout au long du programme de formation. Cela a conduit à la généralisation de l’opérationnalisation des "comités de compétences".

Il ne fait aucun doute que des défis nous attendent étant donné l'ampleur des changements qui toucheront les résidents, le corps professoral, l'administration, les systèmes de technologie de l'information, les hôpitaux et les universités. Un leadership et une gestion du changement solides sont essentiels pendant cette période de transition. Dans l'ensemble, la CBME semble être une nouvelle étape positive de l'amélioration continue de la qualité de notre système d'éducation médicale. Nous encourageons la recherche continue qui documente et comprend l'impact de cette immense transition. Que le voyage commence !

\section{Références}

1. Carraccio C, Wolfsthal SD, Englander R, et al. Shifting paradigms: from Flexner to competencies. Acad Med 2002;77. doi:10.1097/00001888-200205000-00003.

2. Frank JR, Snell LS, Cate OT, et al. Competency-based medical education: theory to practice. Med Teach 2010;32(8):638-45. doi:10.3109/01421 59x.2010.501190.

3. Ten Cate $\mathrm{O}$ and Scheele F. Competency-based postgraduate training: can we bridge the gap between theory and clinical practice? Acad Med 2007;82(6):542-47. doi:10.1097/ACM.0b013e31805559c7.

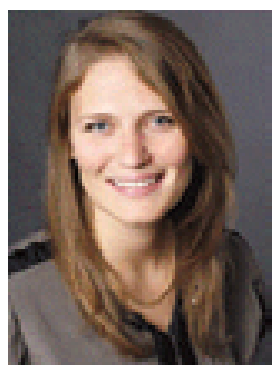

Leslie Martin, MD, MHPE, FRCPC, est professeure adjointe, Division de médecine interne générale, Département de médecine, Université McMaster, Hamilton, ON.

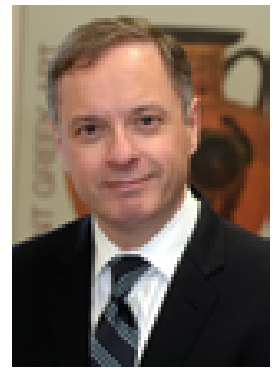

James Douketis M.D., FRCPC Rédacteur en chef, Revue canadienne de médecine interne générale 